\title{
Biomarkers for therapeutic efficacy
}

Brunner, Nils; Nielsen, Kirsten Vang; Offenberg, Hanne Kjær; Sweep, Fred C.G.J.; Martens, John; Foekens, John; Folprecht, Gunnar

Published in:

Connection

Publication date:

2008

Document version

Early version, also known as pre-print

Citation for published version (APA):

Brunner, N., Nielsen, K. V., Offenberg, H. K., Sweep, F. C. G. J., Martens, J., Foekens, J., \& Folprecht, G. (2008). Biomarkers for therapeutic efficacy. Connection, 53-61. 


\section{Biomarkers for Therapeutic Efficacy}

\author{
Nils Brünner ${ }^{1}$, Kirsten Vang Nielsen ${ }^{2}$, Hanne Offenberg ${ }^{1 *}$, \\ Fred C.G.J. Sweep ${ }^{3}$, John Martens ${ }^{4}$, John Foekens ${ }^{4}$, \\ Gunnar Folprecht ${ }^{5}$, and Manfred P. Lutz ${ }^{6}$
}

Professor Nils Brunner, DMSc, MD, got his basic medical education at University of Copenhagen, Denmark. He then trained in internal medicine and medical oncology at hospitals in Greater Copenhagen. In 1987, he went to the National Cancer Institute, NIH, Maryland, USA for one year and then to Lombardi Cancer Research Centre, Georgetown University Hospital, Washington DC for a second year. He got his education in molecular and cellular biology during these two years abroad. Back in Denmark he became employed at the Finsen Laboratory, Rigshospitalet in Copenhagen, where he until 2002 was leading a research group working with translational cancer research within proteinases, their receptors and inhibitors. In 2002 he became full professor at Faculty of Life Sciences, University of Copenhagen. He is leading a research group mainly focusing on non- anti-proteolytic functions of protease inhibitors. He is author of more than 250 publications, many of which deal with translational research on proteinase inhibitors and breast cancer or colorectal cancer. Nils Brünner has a long lasting membership of the EORTC Pathobiology Group, and he served as Chairman of Laboratory Research Division and member of the Executive Board of EORTC 2003-2006.

Kirsten Vang Nielsen is a Senior Principal Scientist at Dako R\&D in Glostrup. Denmark.

Hanne Kjær Offenberg is a research scientist at Novo Nordisk. Previously she was an associate professor at University of Copenhagen, Faculty of Life Sciences/ Dept of Biomedicine.

Fred C.G.J. Sweep is professor of Chemical Endocrinology and head of the department of Chemical Endocrinology at the Radboud University Nijmegen Medical Centre (RUNMC). He is board certified in Clinical Chemistry and Endocrinology by the Netherlands Society for Clinical Chemistry and Laboratory Medicine, and registered as a European Clinical Chemist. He studied Medical Biology at the University of Utrecht (1979-1985), where he also obtained his PhD degree (1989) in Pharmacology. He had his training as a clinical chemist at the RUNMC (1991-1995), where he also completed his training in Endocrinology (1998). Fred Sweep is a reviewer of many journals, member of Editorial boards and has published over 230 papers in peer-reviewed journals (PubMed search code: fred sweep or sweep c or sweep f). Fred Sweep is an active member of many different national and international societies devoted to cancer biomarkers. He is secretary of the PathoBiology Group of the European Organisation for Research and Treatment of Cancer (EORTC) and chairman of the Quality Assurance committee within this group. He also is chairman of the Translation Research Advisory Committee of the EORTC and EORTC board member. In 2006 he chaired the 4th International NCI-EORTC Meeting on Molecular Markers in Cancer, in Atlanta, US. Fred Sweep's department has developed international Quality Assurance programs for steroid hormone receptors and other biomarkers since 1975. In early nineties more than 160 laboratories worldwide participated in these programs. Presently, QA programs are running for large multicentre prospective clinical trials on biomarkers in Europe. His current research interests are focused on development of new antibody based assays for biomarkers in oncology with emphasis on proteases and angiogenesis. Within the field of Endocrinology Sweep's department has a long-standing expertise in thyroid and steroid hormones within special interest in the hypothalamus-pituitary adrenal/gonadal axis and free hormones.

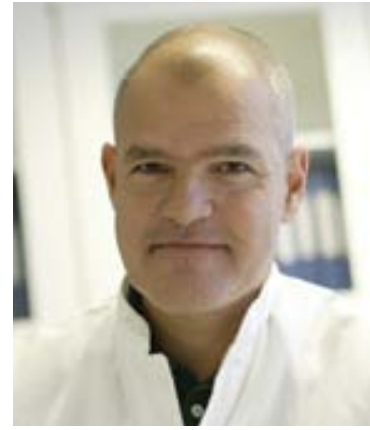

Nils Brünner

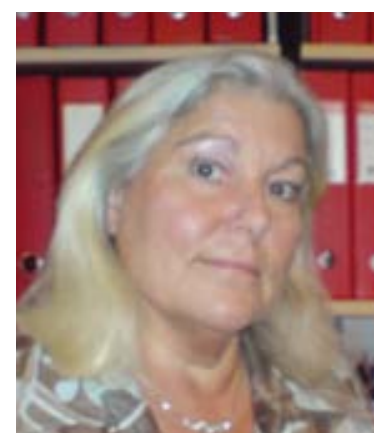

Kirsten Vang Nielsen

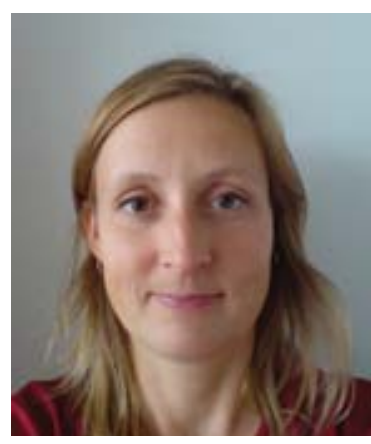

Hanne Offenberg

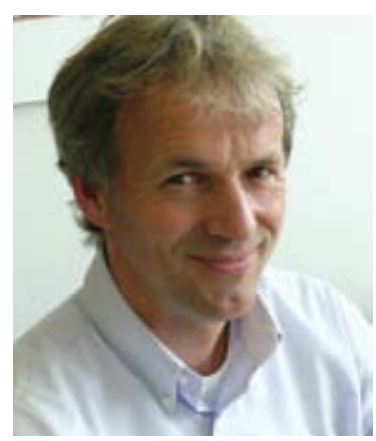

Fred C.G.J. Sweep 
Dr. John W M Martens graduated (1988) and received his PhD (1994) in Molecular Biology at Wageningen University, the Netherlands. After being a post-doc in Molecular Endocrinology, at Erasmus MC, Rotterdam, the Netherlands (1994-1998) and at UCSF in San Francisco, CA, USA (1998-2001), he proceeded his carreer from 2001 onwards as a senior scientist in translational research in breast cancer at the Dept. of Medical Oncology at the Erasmus MC, Rotterdam, the Netherlands. In his current position he focuses on molecular markers related to breast cancer disease progression and has identified epigenetic markers, messenger RNA and miRNA expression signatures linked to breast cancer prognosis, breast cancer dissemination and response to conventional chemotherapy. He is a member of the steering committee of European Organisation for Research and Treatment of Cancer (EORTC)-pathobiology group. He has published various key papers in the field of translational research in breast cancer.

Dr. John A. Foekens is full professor at the Erasmus Medical Center Rotterdam, The Netherlands. He is a biochemist who has been involved in translational cancer research for over 20 years with special interest in prognostic and predictive factors in breast cancer. In 1974 he graduated at the State University of Leiden and in 1982 he obtained his PhD-degree at the Erasmus University Rotterdam. After a post-doc period of 2 years at the Cancer Control Agency of British Columbia in Vancouver, Canada, in 1985 he became the head of the laboratory of Tumor Endocrinology of the Dr. Daniel den Hoed Cancer Center, which now is part of the Erasmus Medical Center Rotterdam. He was appointed full professor in 2008, and currently heads the laboratory of Genomics \& Proteomics of Breast Cancer, section Translational Cancer Genomics \& Proteomics of the department of Medical Oncology. Prof. Foekens has published more than 280 papers and is reviewer of over 30 scientific journals and granting agencies.

Dr. Gunnar Folprecht, MD. Dr. Gunnar Folprecht is head of the outpatient unit for medical oncology at the University Hospital Carl Gustav Carus, Dresden (Germany), which had been selected as one of the four first Centers of Excellence in Germany by the German Cancer Aid. He studied medicine in Würzburg and Dresden, received his training in Medical Oncology and Gastroenterology at the University hospital of Dresden. His scientific focus is clinical research of Colorectal Cancer and multidisciplinary treatment of cancer patients, especially with liver metastases.Dr. Folprecht is a member of several scientific organisations including the European Society for Medical Oncology (ESMO), the American Society of Clinical Oncology (ASCO) and a board member of the European Organisation for Research and Treatment of Cancer (EORTC) Gl group. He is a Coordinator and National Coordinator of several studies in adjuvant treatment or in the treatment of metastatic colorectal Cancer.

\footnotetext{
1. Department of Biomedicine, Faculty of Life Sciences, University of Copenhagen, Denmark. ${ }^{*}$ Research Scientist. Novo Nordisk A/S, Novo Nordisk Park, 2760 Måløv, Denmark).

2. DAKO A/S, Glostrup, Denmark.

3. Department of Chemical Endocrinology, Radboud University Nijmegen Medical Centre, Nijmegen, the Netherlands.

4. Erasmus MC - Daniel der Hoed, Josephine Nefkens Building, Room BE426, Rotterdam, the Netherlands.

5. Medical Department I, University Hospital Dresden, Germany.

6. Department of Medical Gastroenterology, Caritasklinik St. Theresia, Saarbrücken, Germany.

To whom correspondence should be addressed: Nils Brünner, Department of Biomedicine, Faculty of Life Sciences, University of Copenhagen, Ridebanevej 9, 1870 Frederiksberg C, Denmark. Phone: xx45 26144708, e-mail: nbr@life.ku.dk
}

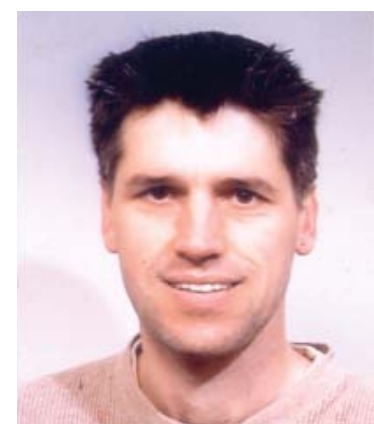

\section{John Martens}

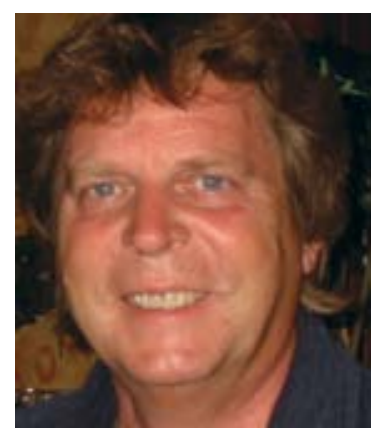

John Foekens

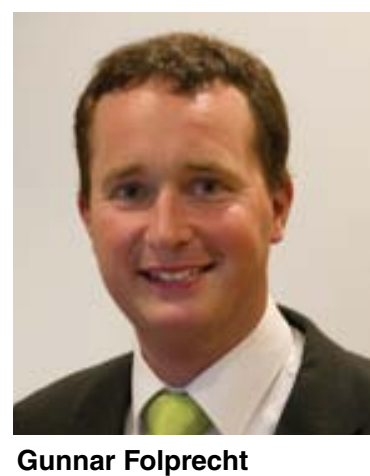

Manfred P. Lutz
(Not available)

-The original version appeared in EJC Vol5 No5 September 2007, Educational Book, ECCO14, p 129-142 


\section{Introduction}

Despite the continuous development of new cancer treatment strategies, including optimization of already known drugs, as well as development of new targeted therapies, too many cancer patients still experience recurrence of their disease with subsequent disease related deaths. It is thus clear that in order to lower the number of deaths, there is an urgent need for the development of more effective treatment strategies including the introduction of new procedures for optimal prediction of response to treatment. Anticancer drug treatment given either alone or in various combinations includes different types of chemotherapy (cytotoxic drugs), endocrine therapy, immunotherapy and recently specified targeted therapies. In most metastatic cancer diseases, the objective response rates (complete and partial responses) to any of these treatment options are far from $100 \%$. This means that a significant number of cancer patients receive chemotherapy with no other effect than systemic toxicity.

While detection of estrogen and progesterone receptors in breast cancer is a well-established routine method to predict objective response to endocrine therapy, there is at present no accepted method to determine whether a cancer patient will benefit from chemotherapy in its broad sense. If such a method existed, it would allow for a tailor-made approach resulting in individualized treatment. This would also imply that for those patients having resistant tumors, such an approach would not only spare them from side effects induced by ineffective chemotherapy, but would also have a major impact on the economics of the health care system in terms of savings of expenses related to otherwise ineffective treatment. Furthermore, it is now clear that predictive molecular assays must be devised before the initiation of clinical trials for new targeted anticancer agents. Use of predictive markers will increase the specificity and usefulness of these drugs and provide a meaningful clinical evaluation in the population of patients most likely to benefit from the treatment. Thus, the field of cancer drug discovery clearly needs to turn greater attention to the problem of identifying responsive/resistant subsets of patients early in the development process and needs to utilize the knowledge obtained through molecular and cellular studies of cancer biology.

\section{DNA Biomarkers}

At the cellular level cancer is a genetic disease and the tumor cells have acquired genetic changes that are responsible for the multistep process that drives the malignant transformation (1). The cancer specific genetic changes may lead to altered mRNA and protein levels and may represent the most important mechanism by which the tumor can permanently acquire new functionality. The acquired specific genetic changes in the cancer cells will however, not be present in the non-malignant cells of the patient. Therefore, it is obvious to exploit the specific genetic changes of the tumor cells as diagnostic, prognostic and especially predictive tools in the management of cancer patients.

The malignant transformation is driven by inactivation of tumor suppressor genes combined with activation of proto-oncogenes. The inactivation of tumor suppressor genes can occur by a variety of mechanisms, including physical deletion, point mutation and/or methylation - all leading to loss of function. Proto-oncogenes, on the other hand, can be activated by amplification, point mutation or structural rearrangements. Minor changes include point mutations and smaller intragenic deletions and duplications and the DNA amount involved ranges from a single base pair to several millions of base pairs. These changes can be studied by sequencing, LOH (loss of heterozygosity) allelic imbalances (AI) and/or PCR based techniques combined with blotting techniques. The methods are precise for the detection of point mutations, but larger deletions and duplications are not revealed. Only by comparing the DNA sequence of the cancer cells with the normal cells of the patient, is it possible to distinguish point mutations from single nucleotide polymorphisms (SNPs). Polymorphism is a normal genetic variation present in the population and thus not directly linked to the cancer. Further, the gene function may be influenced by epigenetic factors, e.g. methylation.

The major genetic changes include large stretches of DNA, from several thousands to millions of base pairs, and may, depending on methodology, be detected as structural rearrangements or copy number changes (CNC). The techniques described above for detection of minor genetic changes cannot reveal the major genetic changes and vice versa. The major genetic changes can be studied by CGH (comparative genomic hybridization), array-CGH and/or FISH (fluorescence in situ hybridization). Using FISH technique, structural rearrangements can be detected as chromosome translocations and the copy number changes are seen as amplifications, deletions, and duplications. FISH methods for detection of these chromosome aberrations were initially developed for cytogenetic specimens (Fig.1) but have later been refined to cut sections of paraffin embedded tissue (Fig. 2). 

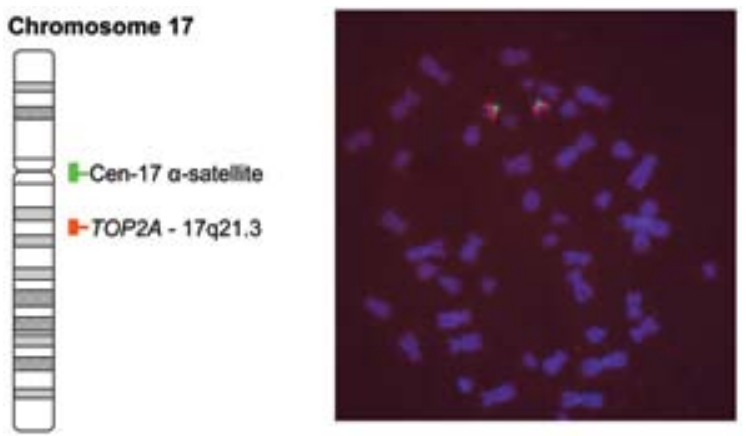

Figure 1. Localisation of TOP2A gene probe in red and centromere 17 reference probe in green schematically on an ideogram (left) and on metaphase spread from normal human blood.

The FISH technique comprises hybridizing fluorescent labeled probes with target DNA of the cancer tissue. The gene directed probe is preferentially 200-400 kilobase pairs in length and includes the cancer related gene and flanking regions. When selecting the genomic clones for diagnostic use, the gene of interest should be located at a position that excludes or minimize presence of flanking genes that potentially could be related to cancer.

Gene copy number changes can be viewed in the nuclei of a tissue and counted directly. A normal cell will contain 2 gene copies and a deviation from this number is indicative of an abnormal cell. However, due to the fact that cut sections of tissue are 4-6 $\mu \mathrm{m}$ in thickness and the nuclei of a cancer cell is often $10 \mu \mathrm{m}$, this simple relationship is not applicable for cut sections. Therefore, a reference probe is added to the probe mix and the ratio between the gene probe (labeled in red) and the reference probe (labeled in green) is scored. Inclusion of a reference probe has further the advantage that gene copy number changes due to polyploidization of the whole genome can be distinguished from amplifications and allows also the detection of deletions. As reference probe, compensating for the ploidy level of the tumor, the centromere of the chromosome that the gene of interest resides on is often used, although it could be any other region of the genome. The signals are scored using a fluorescence microscope equipped with filters that are suited for the red and green fluorochromes. A total of 60 nuclei are normally scored, although alternative counting methods can be used (3). Specific details and variations of the FISH method is described in Nielsen et al. (2).

Predictive DNA biomarkers can be found by either screening cancer cell lines for amplified genes (e.g. HER2), or by studying the gene that is the target for the drug (e.g. TOP2A) or by studying genes involved
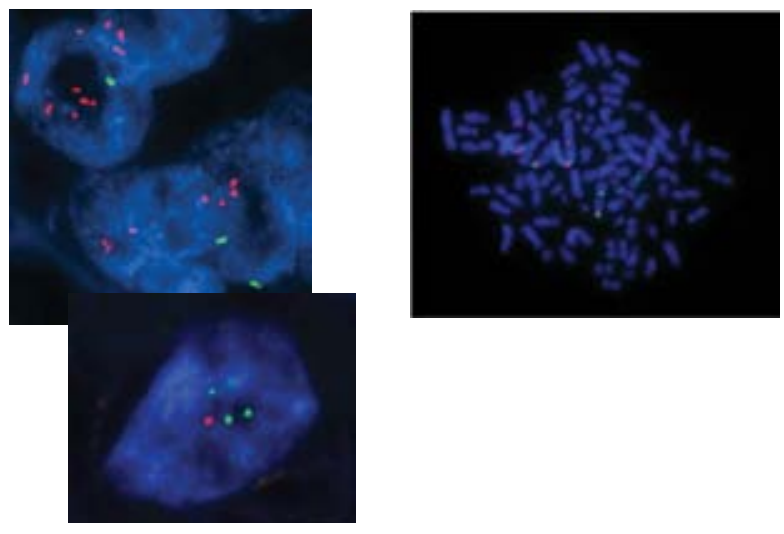

Figure 2. TOP2A gene probe in red and centromere 17 reference probe in green hybridised to breast cancer tissue (left) showing amplification and deletion (middle) and to a metaphase spread of a cell line showing the distribution of the amplified signals onto many different chromosomes (right).

in the pathway of the drug (e.g. EGFR). A number of examples are described below.

One of the most well-established biomarker is the HER2 used for selection of patients for treatment with antibodies directed against HER2. Initially, HER2 (alias of ERBB2) gene amplifications were studied using Southern blotting technique (4), followed by additional information on the mRNA and protein level (5), and eventual leading to the development of a treatment targeting the product of the genetic disorder of the tumor (6). Because the HER2 protein is the therapeutic target, much debate has been devoted to the question about the best use of the different methods, and it is now generally agreed upon (7) that both FISH or immunohistochemistry (IHC) may be used for the assessment of HER2 status in breast cancer. HER2 may, however, be an exceptional case with a very high correlation between the gene copy number studied by FISH and the protein immunoreactivity studied by $I \mathrm{HC}$ and precaution should be taken in generalization from the HER2 case.

Located very close to the HER2 gene on chromosome 17 is the TOP2A gene. The gene codes for topoisomerase Il $\alpha$ which is the target for a group of widely used chemotherapeutic drugs, the anthracyclines. The link between the biomarker and the drug was first described in an in vitro grown cancer cell line (8) having amplification of the TOP2A gene and overexpression of the protein, and initially is was reported that the gene copy number and the mRNA and protein level correlated in this lung cancer cell line (9). Later, studies including samples from breast cancer 
patients, have shown that TOP2A gene aberrations are predictive for the outcome of treatment with anthracyclines $(10,11)$, however a direct correlation between gene copy number and protein amount has not been established $(12,13)$. In contrast to HER2, the DNA and protein measurements are thus not interchangeable in the case of TOP2A and anthracyclines. This relationship is further complicated by the existence of TOP2A deletions.

The epidermal growth factor receptor (EGFR) is amplified or overexpressed in many cancers, including non-small-cell lung cancer and colorectal cancer, and is targeted by EGFR inhibitors. Much effort has been devoted to finding a biomarker that can be used to predict the outcome of treatment with EGFR inhibitors. Initially, IHC, assessing the EGFR protein, was used as a diagnostic tool, and then EGFR mutations were reported, and eventually gene copy number changes were described. Among the various tests developed to predict objective response to EGFR inhibitors, the EGFR-FISH test seems to be the most promising (14), however only very few studies have included and compared all 3 methods (15) and a consensus has not yet been reached regarding the use of biomarkers for selection of therapy with EGFR inhibitors. The consensus is also hampered by the fact that different drugs and different cancer types are being studied using different biomarkers (protein, point mutation or major genetic change), different methodologies for the biomarker and even different cut-offs in defining the discrimination between normal (negative) and abnormal (positive) cases.

The lesson learned from the EGFR puzzle must be that future studies of biomarkers for targeted drugs should be well designed and include pilot studies to determine the methodology and cut-off levels to be used in the confirmatory clinical studies. In addition, if the pilot studies cannot point to the right biomarker, both protein and DNA measurements should be included in the relevant clinical trials. Also, it is important to know that point mutations and amplifications, although both being alterations of the DNA, may not provide the same biological information. Eventually, it may be a combination of 2 or more techniques that should be used to determine the biomarker status.

\section{RNA Biomarkers}

Genetic mutations, genomic losses, amplifications and epigenetic changes including those that control success or failure of chemotherapy, are the driving forces in cancer. The first and currently most easyaccessible reporters of these genetic and epigenetic alterations are the downstream transcripts that are under their control. At present, the most commonly used methodology to study expression of multiple genes in cancer tissue is gene expression microarrays. Current gene expression arrays contain, on a single array, multiple detection probes to all annotated mRNA species of the human genome ( $>25 \mathrm{~K}$ mRNA species). Successful gene expression analysis (16-22) on microarrays and subsequent identification of predictors of disease states involve various steps. In general, fresh-frozen tumor material is used for gene expression analysis since high quality RNA is needed for this to be successful. However, today, it is also possible to extract RNA from formalin-fixed paraffin embedded material. It should be mentioned, however, that this RNA is fragmented and partially chemically modified and therefore currently less useful for microarray discovery studies. This material is, however, highly suited for validations of gene signatures using e.g. quantitative RT-PCR analysis of selected genes.

With regard to the clinical significance of gene expression profiling, the breakthrough came from Stanford Laboratory who identified based on global gene expression five major subgroups in clinical breast cancer specimens (23). Thus, within both the ER-positive (i.e. luminal A and B) and -negative (i.e. basal and normal-like) breast cancers at least two biologically distinct subgroups are present that are different with regard to aggressiveness $(24,25)$ and response to neo-adjuvant paclitaxel containing chemotherapy (26). After these first landmark observations the field has rapidly progressed, and gene signatures associated with endocrine therapy resistance (27), and chemotherapy response (Martens et al., manuscript in preparation) (28-30) have been revealed and predictors of bone and lung relapse uncovered (31-33). For clinical use, the most promising assays are gene expression signatures predicting disease recurrence in breast cancer patients with lymph-node negative disease. Starting with the 70-gene signature predicting disease outcome in young patients $(34,35)$, a wound-healing signature (36), a Nottingham Prognostic Index (37) and genomic grade signature (38) predicting disease recurrence have been identified. A robust 76-gene signature was developed for the prediction of distant metastasis in lymph-node negative patients irrespective of age and hormone receptor status (39). In addition to this, in patients with lymph nodenegative and estrogen receptor-positive disease receiving adjuvant endocrine therapy with tamoxifen, a 21-gene recurrence score (40) and a 2-gene signature, the Interleukin 17B receptor- Homeobox B13 (IL17BR-HOXB13) ratio (41) predicting disease recurrence have been identified. Of these signatures some have been validated $(42,43)$ but only the 70-gene signature from Amsterdam and the 76-gene signature from Rotterdam have been validated in large independent multi-centric cohorts (44-46). This has allowed the initiation of prospective evaluation of the performance of selected prognostic gene signatures in Europe and in the US. Furthermore, for a specific validated multi-gene classifier the FDA recently approved the technology to be used as a prognostic tool in the clinic. 
In conclusion, various predictive gene signatures have been identified while others are being developed. Even though most of them still need independent validation, there is no dispute that predictive gene signatures are likely to be used for therapy decisions in the future.

\section{Protein Biomarkers}

"Proteomics" is a rapidly developing area of cancer research which promises to have an enormous impact on prediction of therapy response in the individual patient. Gel-based and high throughput proteomic technologies, including two-dimensional gel electrophoresis, one- and two-dimensional liquid chromatography, and proteomic microarrays in combination with mass spectrometry (MS) are currently the main tools available to mount a search for cancer protein biomarkers. Proteomic technologies are used for identification of new markers as well as for studying potential differences in the processing of already established protein markers. The hypothesis is that the protein biosynthesis machinery of cancer cells is significantly changed relative to normal cells in relation to the production, degradation and post-translational processing of proteins.

When the protein marker is identified, several different methodologies can be applied when validating the marker in clinical material. The more common methods include immunohistochemical staining of frozen or formalin fixed tissue, and tumor tissue protein extractions followed by immunological methods such as Enzyme-linked Immunosorbent Assay (ELISA) or Radio- Immuno Assay (RIA). The latter methods can also be applied on bodily fluids such as plasma, serum, urine or saliva.

At present a number of proteins, e.g. ER, PgR, ERBB2, and c-kit have proved their value in predicting clinical sensitivity/resistance to targeted cancer therapy. However, no protein measurement is in routine use for prediction of sensitivity/resistance to conventional cytotoxic chemotherapy.

Since most types of chemotherapy induces cell death by activating the apoptosis machinery in the cancer cells, many attempts have been made to relate amount of anti-apoptotic proteins in the cancer cells to degree of sensitivity/resistance to chemotherapy. We have chosen to report on one of these proteins, Tissue Inhibitor of Metalloproteinases type 1 (TIMP-1), since this protein has recently been shown to be involved in cellular protection against apoptosis and thereby resistance to chemotherapy (47). The TIMP1 gene is located on chromosome Xp11.23-11.4 and codes for a soluble $28.5 \mathrm{kDa}$ glycoprotein that consists of 184 amino acids in the mature form. The TIMP-1 protein can be present as a precursor form, as an unbound protein (free TIMP-1) or as a 1:1 stoichiometric complex with proMMP-9 or any of the non membrane-bound active matrix metalloproteinases (MMPs), in the latter case inhibiting the proteolytic activity of the enzymes (for review see Würtz et al (48)).

TIMP-1's inhibition of tissue remodeling processes, such as inflammation, wound healing, and cancer invasion, has mainly been ascribed to its inhibition of MMP mediated proteolytic activity. In contrast, the antiapoptotic function of TIMP-1 has been shown to be MMP-independent. The MMP independent anti-apoptotic pathway was first demonstrated in a study of Burkitt's lymphoma cell lines $(49,50)$. In this study, a positive correlation between TIMP-1 expression and resistance to apoptosis was shown. Addition of recombinant TIMP-1 resulted in inhibition of apoptosis, and addition of anti-TIMP-1 antibodies to neutralize secreted TIMP-1 resulted in a four-fold increase in induction of apoptosis. Of specific interest was that reduced or alkylated TIMP-1, completely devoid of all MMP inhibitory activity, effectively inhibited apoptosis in Burkitt's lymphoma cells $(49,50)$.

Recently, TIMP-1 was shown to interact with CD63 in MCF10A cells (51). CD63 is a member of the tetraspanin family. Of particular interest was that the interaction of TIMP-1 with CD63 inhibited caspase mediated apoptosis. Binding of TIMP-1 to the cell surface initiates a signal transduction cascade through Ras. Ras increases phosphorylation of ERK, and activates the Raf-1/tyrosine kinase/mitogen-activated protein kinase (MAPK) and the phosphoinositide 3-kinase (PI3K) signal pathways leading to stimulation of Cyclin D1 expression. By constitutive activation of focal adhesion kinase (FAK) and/or through the PI3 kinase, which phosphorylates Akt and Bad, TIMP-1 increases the expression of the anti-apoptotic protein $\mathrm{Bcl}-\mathrm{X}_{L}$ and thereby preventing activation of the caspase cascade (52). Fig. 3 shows part of the intracellular signaling induced by TIMP-1 binding to CD63.

In cell culture-based systems, lack of cancer cell TIMP-1 expression results in increased sensitivity towards chemotherapy (47), suggesting a potential use of TIMP-1 tumor tissue measurements in predicting sensitivity/resistance to chemotherapy in clinical cancer. Indeed, we have recently published that women with metastatic breast cancer and high tumor tissue TIMP-1 values show an objective response to antracycline-based chemotherapy of $0 \%$ while patients with low TIMP-1 levels show an objective response rate of $45 \%$ (53).

Similarly, in a study including patients with metastatic colorectal cancer who received 5FU, leucovorin and irinotecan, high plasma TIMP1 levels (dichotomized by the median plasma TIMP-1 value) were indicative of low probability of objective response to chemotherapy (54). This decreased probability of obtaining an objective response to chemotherapy in plasma high TIMP-1 patients was reflected in a significantly decreased time to progression and overall survival of the patients with $62 \%$ of TIMP-1 low patients being alive at 24 months 


\section{TIMP-1 and Apoptosis}

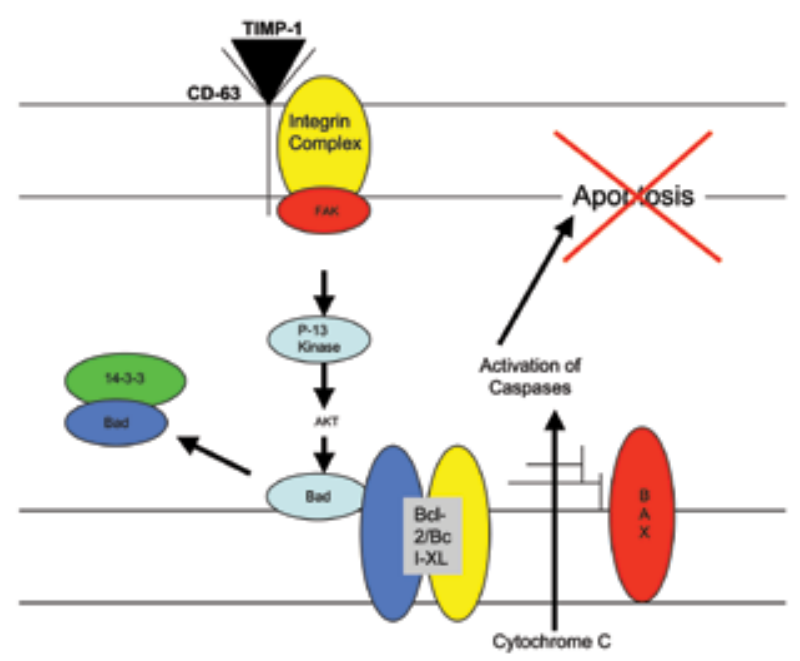

Figure 3. Schematic presentation of part of the intracellular signalling initiated by TIMP-1 binding to CD63

following treatment compared with only $7 \%$ of patients with high plasma TIMP-1 levels being alive at this time point. The Gastrointestinal Cancer Group and the PathoBiology Group of the European Organization for Research and Treatment of Cancer are now in the process of initiating a prospective study validating these findings.

A recent publication (55) describes the association between plasma TIMP-1 levels and response to endocrine therapy in breast cancer. In a cohort of 251 patients with metastatic breast cancer treated with second line endocrine therapy, the objective response rate $(C R+P R+S D)$ was $42 \%$ in patients with low TIMP-1 levels while the objective response rate in TIMP-1 high patients was only $16 \%$. This difference was also reflected in a significant longer time to progression and a significant longer survival of plasma TIMP-1 low patients.

\section{Clinical Validation of Predictive Markers}

In order to have clinical impact, a new predictive marker has to pass a number of analytical and clinical validation steps (56). A major dilemma associated with assays for biomarkers is that various assays may employ different reagents (e.g. antibodies), which may generate non-equivalent test results. Also, variations in reagent preparations, sample processing and the use of different standards may result in discordant test results. Therefore, reagents, assays and procedures should be standardized and the quality of biomarker assay results should be monitored by continuous inter-laboratory proficiency testing of performance. Biomarkers are often used in the clinical setting to provide additional information that will influence clinical decision making, while only few guidelines have been established to inform about how a biomarker should become standard for a certain type of cancer. Hayes et al., 1996 (57) therefore proposed that it is highly necessary to establish standard criteria for evaluation of biomarkers and to standardize the biomarker information for clinical utility. To judge whether these factors have added value over the traditional factors, McGuire et al, 1992 (58) proposed guidelines for evaluating new cancer biomarkers, including a biologic hypothesis for the new factor, adequate sample size, risk of sampling bias, appropriate test system, establishment of cut-off values in a training data-set and confirmation of the observation in a validation data set.

Biomarker assay results often are quite heterogeneous, depending on the composition of the specimen, the way tissue is processed, and design and specificity of an assay and, as important, statistical methods used for evaluation of data. It is of utmost importance to note that uniform handling applies to all of the laboratory steps including use of highly standardized and optimized reagents, tissue collection, storage and processing, the analytical procedures, and subsequent data processing. Within Europe, a multitude of translational multi-centre cancer studies have been co-ordinated by the European Organisation for Research and Treatment of Cancer (EORTC). Within this consortium the PathoBiology Group was established to research and to advice on common, or equivalent, methodologies for biomarker assays and to ensure that appropriate External Quality Assessment (EQA) schemes are applied. As an example, for the past 25 years for ER and PgR, largescale EQA trials, amounting to participating 165 institutions/hospitals from 18 countries, have been carried out and organized by PathoBiology Group $(59,60)$.

When a validated assay is available, the clinical studies can be initiated It is recommended to start analyzing material collected from prior wellcontrolled clinical studies, from which high quality sample material as well as clinical outcome regarding treatment efficacy is available. Following these retrospective studies, the prospective clinical studies can be started. We recommend that the first study is designed as an adjunct to a clinical study in which the primary objective is to test the efficacy of one or more drugs. In such a study, sample collection should be prospective and strictly follow predefined conditions (Standard Operating Procedures) regarding sample collection, storage handling, analyses and data reporting. The next step in the validation is a welldimensioned prospective study where the primary objective is to validate the predictive power of the marker in question. A simple trial design is as follows: Patients are randomized to receive either standard treatment or marker-guided treatment. End-points will be objective response rate (RECIST criteria's), or time to progression and less frequent overall 
survival. This type of clinical studies can only be performed in cancer types for which more than one treatment option exists and is especially helpful if the efficacy of the different treatments is considered equal.

For example, in the majority of patients with metastatic colorectal cancer $(\mathrm{mCRC})$, chemotherapy is the treatment of choice. Survival following chemotherapy of $\mathrm{mCRC}$ has improved substantially over the last years. Whereas survival without chemotherapy was limited to 8.5 months, the first effective drug (5-fluorouracil, 5FU) was shown to increase the median overall survival to 12 months (61). Addition to $5 F U$ of either irinotecan or oxaliplatin increased the efficacy: If one of these drugs is added to 5FU, the response rates rise from approximately $20 \%$ to approximately $50 \%$ with either the combination irinotecan/5FU (FOLFIRI) (62-64) or oxaliplatin/5FU (FOLFOX) (64-66). Irinotecan and oxaliplatin based chemotherapy differs in toxicity profile (diarrhoea and alopecia vs. neurotoxicity), but there is no meaningful difference with regard to the overall efficacy. Because efficacy and overall toxicity are similar in the whole patient population, both regimens are equally used in first line therapy.

One unresolved problem with either of the chemotherapy combinations is that up to $25 \%$ of patients have tumors that are inherently resistant to the chosen chemotherapy schedule (FOLFOX or FOLFIRI) $(67,68,69)$. The current clinical approach selects one treatment regimen over another based on predicted success (and to a lesser degree also toxicity) in large patient cohorts. Using this approach, there necessarily is a large fraction of individual patients who has no benefit whatsoever and may even suffer from the chemotherapy. These patients are unnecessarily exposed to treatment toxicity, and they experience disease progression, which affects the performance status and the capability to tolerate further chemotherapy. In contrast, they might rather profit from an alternative regimen.

In contrast to predicting chemotherapy response, it is at present more important to predict chemotherapy resistance to metastatic colorectal cancer. If chemotherapy resistance to one type of cytotoxic drug could be predicted for individual patients, these patients could already as 1st line treatment receive the other treatment combination and thereby increase their chance of treatment benefit. In addition, such an approach would avoid the costs for approximately 2 months of ineffective treatment (with weekly costs of up to $500 €$ ), and thereby be highly cost-effective. With the currently available evidence, there is no way to predict response or resistance to the given chemotherapeutic treatment in the individual patient, let alone any guidance to select one regimen instead of another one.

Thus, there is a need for research focusing on the identification and development including clinical implementation of predictive markers for each existing chemotherapeutic drug in addition to general markers for chemotherapy sensitivity/resistance. Also, a clear discrimination between diagnostic, prognostic and predictive markers is needed, including statistical methods that can facilitate this separation.

\section{References}

1. Hanahan D, Weinberg RA. The hallmarks of cancer. Cell 2000, 100(1), 57-70.

2. Nielsen KV, Müller S, Poulsen TS, Gabs S, Schonau A. Combined Use of PNA and DNA for Fluorescence In Situ Hybridization (FISH). In: Nielsen PE, editor. Peptide Nucleic Acids: Protocols and Applications. 2 ed. Norfolk: Horizon Bioscience; 2004, 227-60.

3. Olsen KE, Knudsen H, Rasmussen BB et al. Amplification of HER2 and TOP2A and deletion of TOP2A genes in breast cancer investigated by new FISH probes. Acta Oncol 2004, 43, 35-42.

4. Slamon DJ, Clark GM, Wong SG, Levin WJ, Ullich A, McGuire WL. Human breast cancer: correlation of relapse and survival with amplification of the HER-2/neu oncogene. Science 1987, 235, 177-82.

5. Slamon DJ, Godolphin W, Jones LA et al. Studies of the HER-2/neu Proto-oncogene in human breast and ovarian cancer. Science 1989, 244, 707-12.

6. Slamon DJ, Leyland-Jones B, Shak SS et al. Use of chemotherapy plus a monoclonal antibody against HER2 for metastatic breast cancer that overexpresses HER2. Journal of Medicine 2001, 344, 783-92.

7. Wolff $\mathrm{AC}$, Hammond ME, Schwartz JN et al. American Society of Clinical Oncology/College of American Pathologists Guideline Recommendations for Human Epidermal Growth Factor Receptor 2 Testing in Breast Cancer. Arch Pathol Lab Med 2007, 131, 18.
8. Coutts J, Plumb JA, Brown R, Keith WN. Expression of topoisomerase II alpha and beta in an adenocarcinoma cell line carrying amplified topoisomerase II alpha and retinoic acid receptor alpha genes. Br J Cancer 1993 $68,793-800$

9. Withoff S, Keith WN, Knol AJ et al. Selection of a subpopulation with fewer DNA topoisomerase II alpha gene copies in a doxorubicin-resistant cell line panel. $\mathrm{Br}$ J Cancer 1996, 74, 502-7.

10. Knoop A, Knudsen $\mathrm{H}$, Balslev E et al. TOP2A aberrations as predictive and prognostic marker in high-risk breast cancer patients. A randomized DBCG Trial (DBCG89D) In: Journal of Clinical Oncology, ASCO Annual Meeting Proceedings Part I. Vol 24, No. 18S (June 20 Supplement), 2006, 532, 2006.

11. Knoop AS, Knudsen $H$, Balslev $E$ et al. Retrospective analysis of topoisomerase Ila amplifications and deletions as predictive markers in primary breast cancer patients randomly assigned to cyclophosphamide, methotrexate, and fluorouracil or cyclophosphamide, epirubicin, and fluorouracil: Danish Breast Cancer Cooperative Group. $J$ Clin Oncol 2005, 23, 7483-90.

12. Mueller RE, Parkes RK, Andrulis I, O'Malley FP. Amplification of the TOP2A gene does not predict high levels of topoisomerase II alpha protein in human breast tumor samples. Genes Chromosomes Cancer 2004, 39 288-97.
13. Callagy G, Pharoah P, Chin SF et al. Identification and validation of prognostic markers in breast cancer with the complementary use of array-CGH and tissue microarrays. J Pathol 2005, 205, 388-96.

14. Amler LC, Goddard AD, Hillan KJ. Predicting clinical benefit in non-small-cell lung cancer patients treated with epidermal growth factor tyrosine kinase inhibitors. Cold Spring Harb Symp Quant Biol 2005, 70, 483-8.

15. Hirsch FR, Varella-Garcia M, Bunn PA et al. Molecular predictors of outcome with gefitinib in a phase II placebo-controlled study in advanced non-small-cell lung cancer. J Clin Oncol 2006, 24, 5034-42.

16. Tusher VG, Tibshirani R, Chu G. Significance analysis of microarrays applied to the ionizing radiation response. Proc Natl Acad Sci U S A. 2001, 98, 5116-5121.

17. Benjamin $Y$, Hochberg $Y$. Controlling the false discovery rate: A practical and powerful aproach to multiple testing. J. Royal Stat Soc Ser B 1995, 57, 289-300.

18. Cox DR, Oakes D, eds. Analysis of survival data. New York, Chapman and Hall, 1984

19. Johnson R, Wichern DW, eds. Applied multivariate statistical analysis, New Jersey, Prentice Hall, 1998.

20. Vapnik VN, ed. The nature of statistical learning theory. Berlin, Springer-Verlag, 1995.

21. Bishop CM, ed. Neural networks for pattern recognition. Oxford, Clarendon Press, 1995. 
22. Eisen MB, Spellman PT, Brown PO, Botstein D. Cluster analysis and display of genome-wide expression patterns. Proc Natl Acad Sci U S A. 1998, 95, 1486314868.

23. Perou CM, Sorlie T, Eisen MB et al. Molecular portraits of human breast tumours. Nature. 2000, 406, 747-52.

24. Sorlie T, Perou CM, Tibshirani R et al. Gene expression patterns of breast carcinomas distinguish tumor subclasses with clinical implications. Proc Natl Acad Sci U S A. 98 2001, 98, 10869-74.

25. Sorlie T, Tibshirani R, Parker J et al. Repeated observation of breast tumor subtypes in independent gene expression data sets. Proc Natl Acad Sci U S A. 2003, 100, 841823.

26. Rouzier R, Perou CM, Symmans WF et al. Breast cancer molecular subtypes respond differently to preoperative chemotherapy. Clin Cancer Res. 2005, 11, 5678-85

27. Jansen MP, Foekens JA, van Staveren IL et al. Molecular classification of tamoxifen-resistant breast carcinomas by gene expression profiling. J Clin Oncol. 2005, 23, $732-40$.

28. Chang JC, Wooten EC, Tsimelzon A et al. Gene expression profiling for the prediction of therapeutic response to docetaxel in patients with breast cancer. Lancet 2003, 362, 362-69.

29. Hess KR, Anderson K, Symmans WF et al. Pharmacogenomic predictor of sensitivity to preoperative chemotherapy with paclitaxel and fluorouracil, doxorubicin, and cyclophosphamide in breast cancer. $J$ Clin Oncol. 2006, 24, 4236-44.

30. Thuerigen O, Schneeweiss A, Toedt G et al. Gene expression signature predicting pathologic complete response with gemcitabine, epirubicin, and docetaxel in primary breast cancer. J Clin Oncol. 2006, 24, 1839-45.

31. Smid M, Wang Y, Klijn JG et al. Genes associated with breast cancer metastatic to bone. J Clin Oncol. 2006, 24. 2261-67.

32. Minn AJ, Gupta GP, Siegel PM et al. Genes that mediate breast cancer metastasis to lung. Nature. 2005, 436, 518-24.

33. Minn AJ, Kang Y, Serganova I et al. Distinct organspecific metastatic potential of individual breast cancer cells and primary tumors. J Clin Invest. 2005, 115, $44-55$

34. Van 't Veer LJ, Dai H, van de Vijver MJ et al. Gene expression profiling predicts clinical outcome of breast cancer. Nature 2002, 415, 530-36.

35. van de Vijver MJ, He YD, van't Veer LJ et al. A geneexpression signature as a predictor of survival in breast cancer. N Engl J Med 2002, 347, 1999-2009.

36. Chang HY, Nuyten DS, Sneddon JB et al. Robustness, scalability, and integration of a wound-response gene expression signature in predicting breast cancer survival. Proc Natl Acad Sci U S A 2005, 102, 3738-43.

37. Yu K, Lee CH, Tan PH, Hong GS, Wee SB, Wong CY, Tan P. A molecular signature of the Nottingham prognostic index in breast cancer. Cancer Res 2004, 64, 2962-68.

38. Sotiriou C, Wirapati P, Loi S et al. Gene expression profiling in breast cancer: understanding the molecular basis of histologic grade to improve prognosis. J Natl Cancer Inst 2006, 98, 262-72.

39. Wang Y, Klijn JG, Zhang Y et al. Gene-expression profiles to predict distant metastasis of lymph-node-negative primary breast cancer. Lancet 2005, 365, 671-79.
40. Paik S, Shak S, Tang G et al. A multigene assay to predict recurrence of tamoxifen-treated, node-negative breas cancer. N Engl J Med 2004, 351, 2817-26.

41. Ma XJ, Wang Z, Ryan PD et al. A two-gene expression ratio predicts clinical outcome in breast cancer patients treated with tamoxifen. Cancer Cell 2004, 5, 607-16.

42. Goetz MP, Suman VJ, Ingle JN et al. A two-gene expression ratio of homeobox 13 and interleukin-17B receptor for prediction of recurrence and survival in women receiving adjuvant tamoxifen. Clin Cancer Res 2006, 12, 2080-87.

43. MaXJ, Hilsenbeck SG, Wang Wet al. The HOXB13:IL17BR expression index is a prognostic factor in early-stage breast cancer. J Clin Oncol 2006, 24, 4611-19.

44. Foekens JA, Atkins $D$, Zhang $Y$ et al. Multicenter validation of a gene expression-based prognostic signature in lymph node-negative primary breast cancer. J Clin Oncol 2006, 24, 1665-71.

45. Buyse M, Loi S, van't Veer $L$ et al. Validation and clinica utility of a 70-gene prognostic signature for women with node-negative breast cancer. J Natl Cancer Inst 2006, $98,1183-92$

46. Desmedt C, Piette F, Loi S et al. Strong time-dependency of the 76-gene prognostic signature for node-negative breast cancer patients in the TRANSBIG multi-center independent validation series. Clin Cancer Res. (in press)

47. Davidsen ML, Wurtz SO, Romer MU et al TIMP-1 gene deficiency increases tumour cell sensitivity to chemotherapy-induced apoptosis. Br J Cancer 2006, 95, 1114-20.

48. Würtz SO, Schrohl AS, Sorensen NM et al. Tissue inhibitor of metalloproteinases-1 in breast cancer. Endocr Relat Cancer 2005, 12, 215-27.

49. Guedez L, Stetler-Stevenson WG et al. In vitro suppression of programmed cell death of $B$ cells by tissue inhibitor of metalloproteinases-1. J Clin Invest 1998, 102, 2002-10

50. Guedez L, Courtemanch L, Stetler-Stevenson M. Tissue inhibitor of metalloproteinase (TIMP)-1 induces differentiation and an antiapoptotic phenotype in germinal center B cells. Blood 1998, 92, 1342-49.

51. Jung KK, Liu XW, Chirco R, Fridman R, Kim HR Identification of $\mathrm{CD} 63$ as a tissue inhibitor of metalloproteinase-1 interacting cell surface protein. EMBO J 2006, 25, 3934-42.

52. Liu XW, Bernardo MM, Fridman R, Kim HR. Tissue inhibitor of metalloproteinase-1 protects human breast epithelial cells against intrinsic apoptotic cell death via the focal adhesion kinase/phosphatidylinositol 3-kinase and MAPK signaling pathway. J Biol Chem 2003, 278, 40364-72.

53. Schrohl AS, Meijer-van Gelder ME, Holten-Andersen MN et al. Primary tumor levels of tissue inhibitor of metalloproteinases- 1 are predictive of resistance to chemotherapy in patients with metastatic breast cancer. Clin Cancer Res 2006, 12, 7054-8.

54. Sørensen N, Byström P, Christensen IJ et al. Plasma Tissue Inhibitor of Metalloproteinases-1 (TIMP-1) is significantly associated with objective response and survival in patients with metastatic colorectal cancer receiving first line treatment with irinotecan in combination with 5FU and Folinic acid. Clin Cancer Res 2007, in press.

55. Lipton A, Ali SM, Leitzel K et al. Elevated plasma tissue inhibitor of metalloproteinase-1 level predicts decreased response and survival in metastatic breast cancer Cancer 2007, 109, 1933-9.
56. Sweep FCGJ, Fritche H, Gion M, Klee GG, Schmitt M Considerations on development, validation, application and quality control of immuno(metric) biomarker assays in clinical cancer research. An EORTC-NCl working group report. Int J Oncol 2003, 23, 1715-26.

57. Hayes DF, Bast RC, Desc CE, Fritsche $\mathrm{H}$, Kemeny NE, Jessup JM, Locker GY, Macdonald JS, Mennel RG, Norton L, Ravdin P, Taube S and Winn RJ: Tumor marker utility grading system: a framework to evaluate clinical utility of tumor markers. J Natl Cancer Inst 1996, 88, 1456-66.

58. McGuire WL and Clark GM: Prognostic factors and treatment decisions in axillary-node-negative breast cancer. N Engl J Med 1992, 326, 1756-61.

59. Geurts-Moespot J, Leake R, Benraad ThJ and Sweep CGJ: Twenty years of experience with the steroid receptor External Quality Assessment program - the paradigm for tumour biomarker EQA studies (review). Int J Oncol 2000, 17, 13-22.

60. Sweep CGJ and Geurts-Moespot J: EORTC external quality assurance program for $\mathrm{ER}$ and $\mathrm{PgR}$ measurements: trial 1998/1999. Int J Biol Markers 2000 $15,62-9$.

61. Simmonds PC. Palliative chemotherapy for advanced colorectal cancer: systematic review and meta-analysis. Colorectal Cancer Collaborative Group. BMJ 2000, 321 531-5.

62. Saltz LB, Cox JV, Blanke C, Rosen LS, Fehrenbacher $L$, Moore MJ et al. Irinotecan plus fluorouracil and leucovorin for metastatic colorectal cancer. Irinotecan Study Group. N Engl J Med 2000, 343,905-14.

63. Douillard JY, Cunningham D, Roth AD, Navarro $M$ James RD, Karasek P et al. Irinotecan combined with fluorouracil compared with fluorouracil alone as first-line treatment for metastatic colorectal cancer: a multicentre randomised trial. Lancet 2000, 355,1041-7.

64. Köhne CH, Van Cutsem E, Wils J, Bokemeyer C, El-Serafi M, Lutz MP et al. Phase III Study of Weekly High-dose Infusional 5-Fluorouracil Plus Folinic Acid With or Without Irinotecan in Patients With Metastatic Colorectal Cancer. EORTC Gastrointestinal Group Study 40986. J Clin Oncol 2005, 23, 4856-65

65. de Gramont A, Figer A, Seymour M, Homerin M, Hmiss A, Cassidy $\mathrm{J}$ et al. Leucovorin and fluorouracil with or without oxaliplatin as first-line treatment in advanced colorectal cancer. J Clin Oncol 2000,18, 2938-47.

66. Giacchetti S, Perpoint B, Zidani R, Le Bail N, Faggiuolo $\mathrm{R}$, Focan $\mathrm{C}$ et al. Phase III multicenter randomized trial of oxaliplatin added to chronomodulated fluorouracilleucovorin as first-line treatment of metastatic colorecta cancer. J Clin Oncol 2000,18,136-47.

67. Fruehauf JP, Alberts DS. Assay-assisted treatmen selection for women with breast or ovarian cancer. Recent Results Cancer Res, 2003, 161,126-45.

68. Alberts DS, Dorr RT, Wunz TP et al. In vitro cytotoxicity against fresh human tumors and P388 leukemia predict the differential in vivo activity of a series of anthracene anticancer drugs. Anticancer Drugs, 1991, 2, 69-77.

69. Tournigand $\mathrm{C}$, Andre $\mathrm{T}$, Achille $\mathrm{E}$, Lledo G, Flesh M, Mery-Mignard D et al. FOLFIRI Followed by FOLFOX6 or the Reverse Sequence in Advanced Colorectal Cancer: A Randomized GERCOR Study. J Clin Oncol 2004,15 229-37. 